\title{
Rare case of solitary plasmacytoma of the skull in a young male patient
}

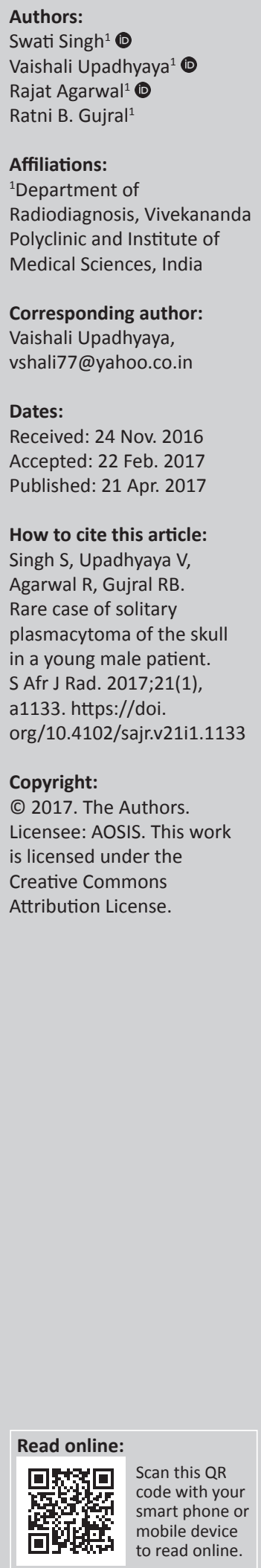

Solitary plasmacytoma of bone without signs of multiple myeloma is a rare entity. It usually presents as an osteolytic lesion in the axial skeleton of an elderly patient. Here, we report a case of solitary plasmacytoma in the skull of a young male patient which emphasises the need to consider it in the differential diagnosis of a destructive calvarial mass lesion even in this age group.

\section{Introduction}

Plasma cell neoplasms are a group of disorders characterised by the neoplastic proliferation of a single clone of plasma cells. Solitary plasmacytoma of bone (SPB) also known as osseous plasmacytoma is a rare form constituting less than $5 \%$ of all cases of plasma cell disorders. It presents at the median age of 55 years. ${ }^{1}$ Being the disease of older adults, the occurrence of SPB in a young individual is extremely rare, with only about $1.1 \%$ of cases occurring in individuals younger than 30 years of age. ${ }^{2}$ We would like to report such a case of SPB in the skull of a young male patient.

\section{Case report}

A 25-year-old male patient presented with a 6-month history of a gradually progressive, painless swelling at the back of the head associated with headache. On examination, there was a firm, nontender, sub- cutaneous swelling in the left parieto-occipital region with normal overlying skin. No neurological abnormality was evident. Haemoglobin, blood counts, blood urea, serum creatinine and electrolytesincluding serumcalcium were doneand found tobenormal.Immunoelectrophoresis for serum proteins revealed normal levels of immunoglobulins. Urinalysis for Bence-Jones proteins showed no abnormal findings. Bone marrow examination was done which did not reveal any signs of systemic myelomatosis.

X-rays of the skull showed a well-defined lytic lesion in the left parietal bone with an associated large soft tissue component. No marginal sclerosis or periosteal reaction was noted (Figure 1a and $1 \mathrm{~b}$ ). Non-contrast computed tomography (NCCT) revealed a well-defined heterogeneous hyperdense mass lesion centred on the left parietal bone causing bone destruction, compressing the adjacent brain parenchyma and bulging into the scalp. A few small bony fragments were seen at the periphery of the lesion (Figure 2). Magnetic resonance imaging (MRI) of the brain with contrast demonstrated a well-defined heterogeneously enhancing soft tissue mass lesion measuring $8.0 \mathrm{~cm}$ $\times 7.9 \mathrm{~cm} \times 6.8 \mathrm{~cm}$ causing destruction of the left parietal bone and bulging into the scalp. Some flow voids were seen with the mass. No restricted diffusion or surrounding oedema was noted. There was mass effect with compression of adjacent brain parenchyma and mild midline shift to the right side. The mass was compressing adjacent superior sagittal sinus but not invading it (Figures 3, 4, 5 and 6). Skeletal survey of the body revealed no other lytic lesions anywhere in the body.

The patient underwent left parieto-occipital craniotomy with gross total excision of the mass. Histopathological examination revealed a plasmacytoma with massive amyloid deposition.

\section{Discussion}

Solitary plasmacytoma of bone without signs of multiple myeloma is a rare entity. The diagnosis is based on the presence of radiologically solitary bone lesion; histological evidence of tumour consisting of monoclonal plasma cells; fewer than 5\% plasma cells in bone marrow; less than $2.0 \mathrm{~g} / \mathrm{dL}$ monoclonal protein (M protein) in the serum when it is present; negative urine test for Bence-Jones protein with no evidence of hyperglobulinemia, hypercalcaemia or anaemia. ${ }^{3}$

Solitary plasmacytoma of bone mostly occurs in axial skeleton, such as skull and vertebra. It shows male preponderance and is extremely rare in patients less than 30 years of age. ${ }^{4}$ 


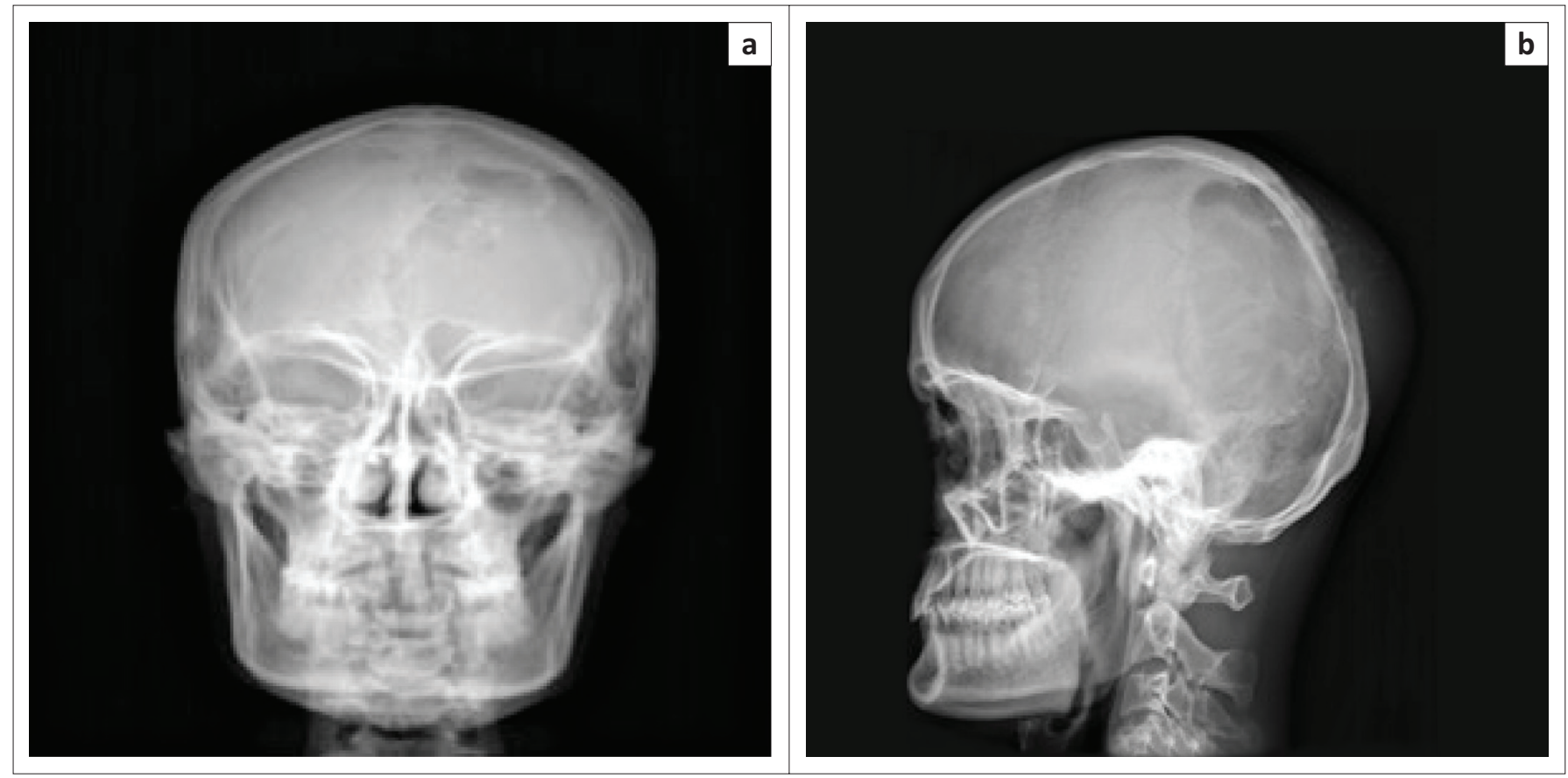

FIGURE 1: (a) X-ray of the skull PA view shows a well-defined lytic lesion with irregular margins in the left parietal bone and (b) X-ray of the skull lateral view shows the associated soft tissue component. No sclerosis or periosteal reaction is seen.

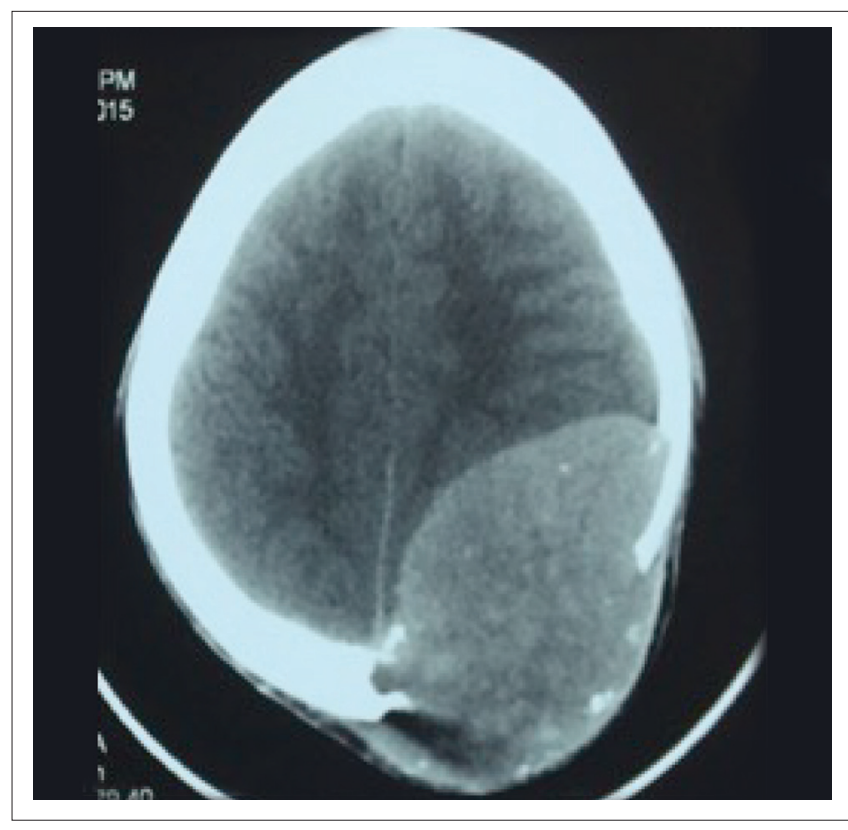

FIGURE 2: Non-enhanced CT scan in axial plane shows a heterogeneous mass lesion in the left parietal bone causing bone destruction with compression of adjacent brain parenchyma and extension into the scalp.

On radiography, SPB presents as a well-defined osteolytic lesion with clear margins and narrow zone of transition. There is no bony sclerosis or periosteal reaction. The lesion is hyperdense on NCCT and shows marked homogenous enhancement with contrast. Small bony fragments may be seen at the periphery. On MRI, SPB appears isointense on T1-weighted images and hyperintense on T2-weighted images. Intratumoural flow voids have been described. The lesion shows dense but heterogeneous enhancement with contrast. ${ }^{3,5,6}$

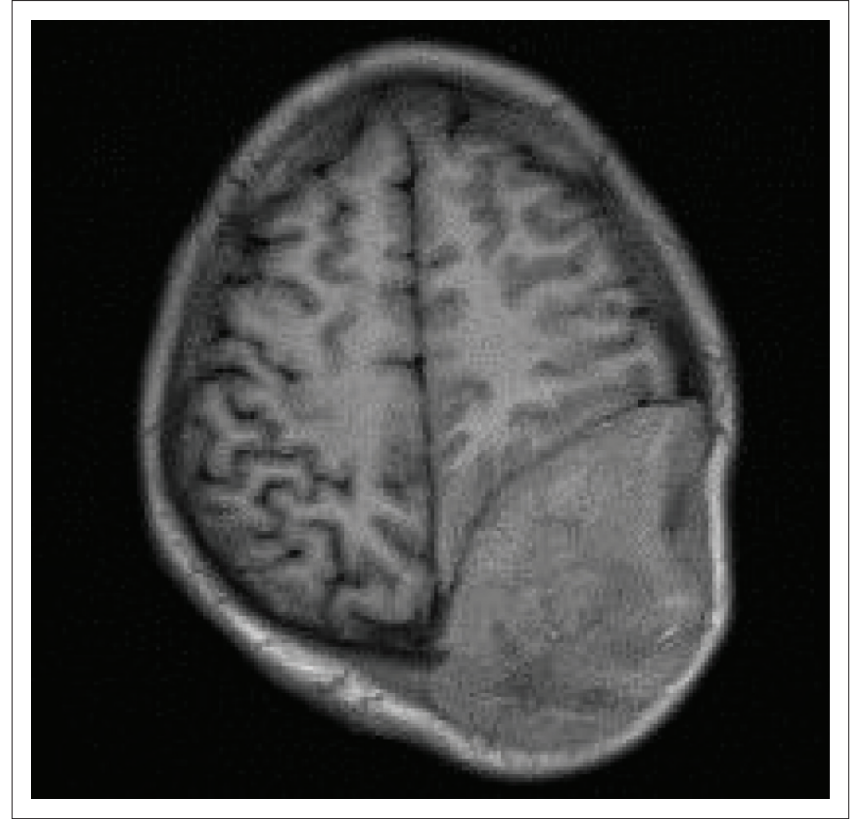

FIGURE 3: Axial T1-weighted MR image shows a heterogeneous mass lesion in left parietal bone causing bone destruction.

Although plasmacytoma was part of the radiological differential in our case, it was considered unlikely taking into account its extreme rarity in young patients.

The other radiological differentials that were considered included osteosarcoma, metastasis, eosinophilic granuloma and bone lymphoma.

Osteosarcoma is the most common primary bone malignancy and frequently occurs in adolescence. Osteosarcoma usually involves long bones with primary calvarial involvement 


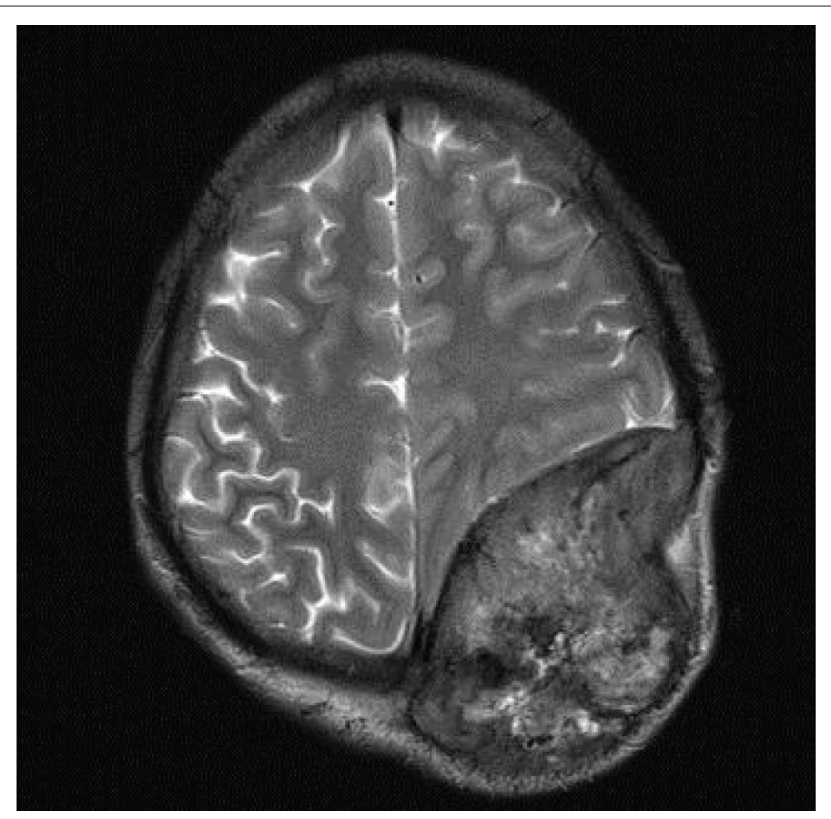

FIGURE 4: Axial T2-weighted MR image showing the mass with lack of edema in adjacent brain parenchyma.

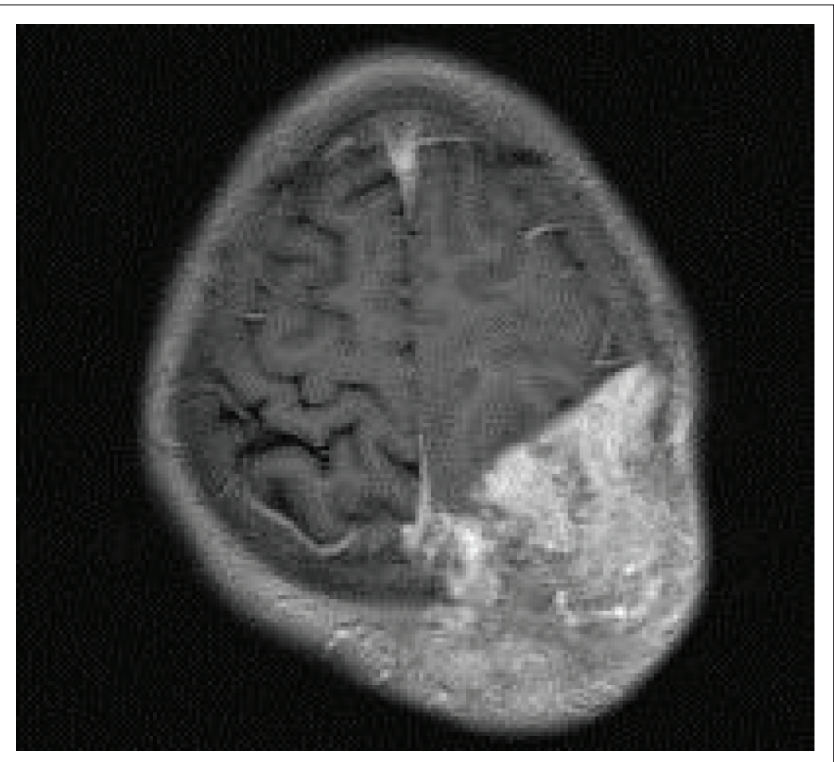

FIGURE 5: Contrast enhanced T1-weighted axial MR image showing intense heterogenous enhancement of the mass lesion.

being rare. In our case, the lesion could have been an osteosarcoma but lack of matrix mineralisation and periosteal reaction were points against this diagnosis.

Calvarial metastases are usually blood-borne metastases arising secondarily to lung, kidney, thyroid and gastro-intestinal carcinomas; sarcomas; and malignant melanomas. These are often small and multiple but can be single and large with regular or irregular margins. In our case, no primary tumour was detected elsewhere making metastasis an unlikely possibility.

Eosinophilic granuloma is usually seen in children and young adults with skull as a predilection site. In our patient,

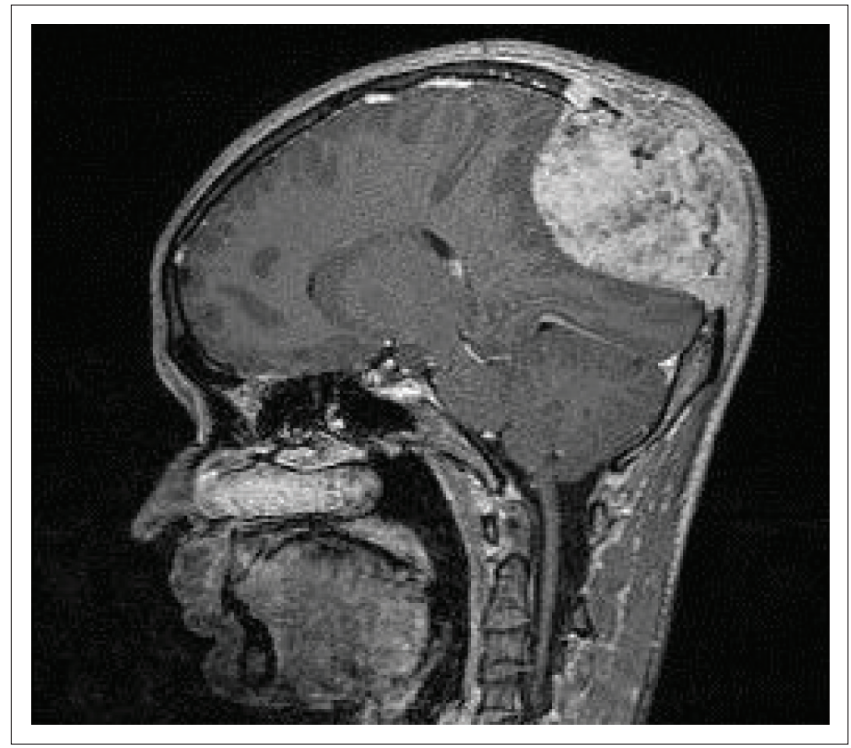

FIGURE 6: Contrast enhanced sagittal T1-weighted MR image also shows the mass lesion with lack of infiltration of adjacent brain parenchyma.

the tumour was a large, well-demarcated lytic mass lesion, and typical radiological findings such as bevelled edges and button sequestrum were absent.

Primary lymphoma of the skull is extremely rare. It shows permeative or moth eaten type of bone destruction associated with soft tissue component. However, this was not consistent with the pattern of bone destruction seen in our case where a large well-defined lytic lesion was found in the left parietal bone.

\section{Conclusion}

Although typically a disease of older adults, this case report emphasises the need to consider the possibility of SPB in the differential diagnosis of a large calvarial mass lesion causing bone destruction even in younger patients.

\section{Acknowledgements Competing interests}

The authors declare that they have no financial or personal relationships that may have inappropriately influenced them in writing this article.

\section{Authors' contributions}

S.S. was responsible for literature compilation. V.U. was responsible for image acquisition, guiding and editing the manuscript. R.A. was responsible for image editing and typesetting. The idea and guidance regarding this article was given by R.B.G.

\section{References}

1. Dimopoulas MA, Moulopoulos LA, Maniatis A, Alexanian R. Solitary plasmacytoma of bone and asymptomatic multiple myeloma. Blood. 2000;96:2037-2044.

2. Jawad MU, Scully SP. Skeletal Plasmaytoma: Progression of disease and impact of local treatment; an analysis of seer database. J Hematol Oncol. 2009;2:41. https:// doi.org/10.1186/1756-8722-2-41 
3. Tanaka M, Shibui S, Nomura K, Nakanishi Y. Solitary plasmacytoma of the skull: A case report. Jpn J Clin Oncol. 1998;28(10):626-630. https://doi.org/10.1093/jjco/28.10.626

4. Kilciksiz S, Celik OK, Agaoglu FY, Haydaroglu A. A review for solitary plasmacytoma of bone and extramedullary plasmacytoma. Scientific World J. 2012;2012:895765. https://doi.org/10.1100/2012/895765
5. Okamoto K, Ito J, Funusawa T, et al. Solitary plasmacytoma of the occipital bone: A report of two cases. Eur Radiol. 1997;7:503-506. https://doi.org/10.1007/ s003300050192

6. Mahale A, Ullal S, Thiagarajan D, Das S. Plasmacytoma of the base of skull - A case report. Indian J Med Paediatr Oncol. 2007;28(3):34-37. 